\title{
A STOCHASTIC SIS INFECTION MODEL INCORPORATING INDIRECT TRANSMISSION
}

\author{
DAMIAN CLANCY, ${ }^{*}$ The University of Liverpool
}

\begin{abstract}
We describe a stochastic susceptible-infective-susceptible (SIS) model for transmission of infectious disease through a population, incorporating both direct host-host transmission and indirect transmission via free-living infectious stages (e.g. environmental bacteria). Existence of a quasi-stationary distribution conditional upon nonextinction of infection is established. A bivariate Ornstein-Uhlenbeck approximation is used to investigate the long-term behaviour of the process conditional upon nonextinction of infection. We show that indirect transmission leads to lower variability in the number of infected hosts present in quasi-stationarity and, consequently, to a greater tendency of infection to persist, compared with a model with direct transmission only and the same average individual infectivity. Some numerical work illustrating these results is presented.
\end{abstract}

Keywords: Quasi-stationary distribution; normal approximation; free-living infectious stage; piecewise-deterministic Markov process

2000 Mathematics Subject Classification: Primary 60J27

Secondary 92D30

\section{Introduction}

In stochastic modelling of infectious disease transmission, the most commonly considered route of infection is direct transmission from infected hosts to susceptible hosts. One widely studied alternative mechanism is the host-vector route appropriate, for example, for malaria, where infection passes from the infected host to an intermediate vector (mosquito) and can then be passed on from the vector to susceptible hosts. Another alternative, suggested by Anderson and May [1], is the existence of free-living infectious stages. That is, the microparasites that cause infection may be able to exist outside the host, so that after being shed (for instance in faeces), the free-living infectious stages survive in the environment for a time and may be consumed by susceptible hosts, potentially infecting them.

In [1] the interest was in infections of invertebrate hosts, and the model with free-living infectious stages (model G of [1]) did not allow for any other means of transmission. More recently, Turner et al. [15] formulated and analyzed a deterministic (ordinary differential equation) model for the spread of the Escherichia coli $\mathrm{O} 157$ bacterial infection amongst dairy cattle, incorporating both direct host-host transmission and indirect transmission via free-living environmental bacteria, as well as vertical transmission. Their model is designed to represent transmission within a typical UK dairy herd and incorporates a variety of realistic features, including demographic processes and herd structure as well as multiple transmission routes. One reason why the free-living infectious stages are explicitly modelled in [15] is that, after an

Received 9 February 2005; revision received 8 March 2005.

* Postal address: Department of Mathematical Sciences, The University of Liverpool, Liverpool L69 7ZL, UK. Email address: d.clancy@liv.ac.uk 
initial major epidemic followed by apparent disease extinction, one or more secondary outbreaks may occur. While it is possible that later outbreaks are caused by reinfection by external sources, another possibility is that, after infection has died out from the host population, it may persist in the environment in the form of bacteria shed in the faeces of infected animals, and may later be transmitted back from the environment to the hosts.

In this paper, we focus upon the role of indirect versus direct transmission, looking at inherently stochastic features that are not well described by the deterministic models of [1] and [15]. Specifically, we consider an extension of the classical susceptible-infected-susceptible (SIS) logistic infection model, defined as follows.

Consider a closed population of size $N$, which at time $t \geq 0$ consists of $S(t)$ susceptible and $I(t)$ infective host individuals, and associated with which is a population of $W(t)$ freeliving infectious stages. When a host individual becomes infected, it remains so for a time that is exponentially distributed with mean $\gamma^{-1}$, before returning to the susceptible class. During its infectious period, each infective host makes contacts at the points of a homogeneous Poisson process of rate $\beta$. Each contact is with an individual chosen uniformly at random from the host population, and the susceptible individuals thus contacted then themselves become infective. During its infectious period, the infective host also sheds infectious stages into the environment at the points of a homogeneous Poisson process of rate $\lambda$. Each host, whether infective or susceptible, consumes free-living infectious stages from the environment according to a Poisson process of rate $(v / N) W(t)$; whenever a susceptible host consumes a free-living infectious stage, with probability $p$ the susceptible host becomes infected. Finally, free-living infectious stages, if not consumed, survive in the environment for a time that is exponentially distributed with mean $\mu^{-1}$. We refer to this as the SIS/W model.

More precisely, we suppose that the process $\{(I(t), W(t)): t \geq 0\}$ is a continuous-time Markov chain on the state space $\left\{(i, w) \in\{0,1, \ldots, N\} \times \mathbb{Z}^{+}\right\}$with infinitesimal transition rates

$$
\begin{aligned}
q_{(i, w),(i+1, w)} & =(\beta / N) i(N-i), \\
q_{(i, w),(i+1, w-1)} & =(p v / N) w(N-i), \\
q_{(i, w),(i-1, w)} & =\gamma i, \\
q_{(i, w),(i, w+1)} & =\lambda i, \\
q_{(i, w),(i, w-1)} & =\mu w+(v / N)(1-p) w(N-i)+(v / N) w i,
\end{aligned}
$$

for some constants $\beta, \gamma, \lambda, \mu, v \geq 0$, and $p, 0 \leq p \leq 1$ (all other transition rates being zero). We further suppose that $S(t)=N-I(t)$.

In the case $p v=0$, the process $I(t)$ reduces to the classical SIS infection model of Weiss and Dishon [16]. The classical SIS model, and likewise our extended SIS/W model, can model either short-term minor outbreaks of infection or long-term endemic behaviour, depending upon parameter values. We shall be concerned mainly with the endemic case and, in particular, the long-term behaviour of the process prior to eventual disease extinction. Long-term (quasi-stationary) behaviour of the classical SIS model was analyzed in some detail in Nåsell [10], [11]. Our aim here being to study the effect of indirect versus direct transmission in endemic behaviour, the model defined by (1.1)-(1.5) is intended to be the simplest possible model incorporating these features. A more complex stochastic SIS/W model, incorporating demographic processes and also vertical transmission of infection, was investigated by Xiao et al. [17] by simulation and by numerical investigation of a normal approximation similar to that of Section 3 below. 
The basic reproduction ratio $R_{0}$ for our SIS/W model (being the average number of new infections directly attributable to a typical infective individual in an otherwise wholly susceptible population) is given by

$$
R_{0}=\frac{\beta}{\gamma}+\frac{p \nu \lambda}{\gamma(\mu+v)} .
$$

A deterministic version of our model can be defined by

$$
\begin{aligned}
\frac{\mathrm{d} \hat{\imath}}{\mathrm{d} t} & =\beta \hat{\imath}(1-\hat{\imath})+p \nu \hat{w}(1-\hat{\imath})-\gamma \hat{\imath}, \\
\frac{\mathrm{d} \hat{w}}{\mathrm{~d} t} & =\lambda \hat{\imath}-(\mu+v) \hat{w},
\end{aligned}
$$

where $N \hat{\imath}(t)$ and $N \hat{w}(t)$ respectively correspond to the numbers of infected hosts and freeliving infectious stages present at time $t \geq 0$ in a population of $N$ hosts. The deterministic system (1.6) has equilibrium points at $(\hat{\imath}, \hat{w})=(0,0)$ and

$$
\left(\hat{\imath}^{*}, \hat{w}^{*}\right)=\frac{R_{0}-1}{R_{0}}\left(1, \frac{\lambda}{\mu+v}\right) .
$$

If $R_{0} \leq 1$ then the system is below threshold; the only equilibrium point $(\hat{\imath}, \hat{w})$ with $\hat{\imath}, \hat{w} \geq 0$ is the disease-free equilbrium $(0,0)$, and this is a stable point. If $R_{0}>1$ then the system is above threshold, there is a second equilibrium with $\hat{\imath}, \hat{w} \geq 0$ at $\left(\hat{\imath}^{*}, \hat{w}^{*}\right)$, and this endemic equilibrium point is stable whereas the disease-free equilibrium $(0,0)$ is unstable.

\section{Existence of a quasi-stationary distribution}

The state space of our stochastic SIS/W model consists of a single absorbing state $(0,0)$ together with the irreducible class $C=\left\{(i, w) \in\{0,1, \ldots, N\} \times \mathbb{Z}^{+}\right\} \backslash\{(0,0)\}$, provided that $\beta, \gamma, \lambda, \mu, v, p, N>0$. Our interest is in the long-term behaviour of the infection process prior to eventual extinction, that is, in the limiting conditional distribution $m_{i w}$ defined by

$$
m_{i w}=\lim _{t \rightarrow \infty} \operatorname{Pr}((I(t), W(t))=(i, w) \mid(I(t), W(t)) \in C), \quad(i, w) \in C,
$$

provided such a distribution $m_{i w}$ exists. Such limiting conditional distributions are closely connected to quasi-stationary distributions. A probability distribution $\pi_{i w}$ defined on $C$ is said to be a quasi-stationary distribution if, given $\pi_{i w}$ as the initial distribution of the process, we have $\operatorname{Pr}((I(t), W(t))=(i, w) \mid(I(t), W(t)) \in C)=\pi_{i w}$ for all $t \geq 0$. Every limiting conditional distribution is a quasi-stationary distribution; while the converse usually holds, counterexamples can be constructed (see Pakes [13] and references therein).

General results regarding the existence of both quasi-stationary and limiting conditional distributions for processes with infinite state space are rather scarce, although certain onedimensional processes, notably the birth-and-death process, have been studied extensively (see Kijima et al. [7] and references therein). For two-dimensional population processes, such as our SIS/W model, it is often necessary to simply assume the existence of the limiting conditional distribution before considering methods for its approximation (see, for example, Nåsell [12] and Clancy et al. [4]). In the present case, our SIS/W process may be treated using the results of Ferrari et al. [6]. The details are as follows.

First of all, the transition rate matrix defined by (1.1)-(1.5) is clearly conservative and, since the rates at which new infective hosts and free-living infectious stages are created 
(given by (1.1)-(1.2) and (1.4), respectively) are bounded, it is immediate from Proposition 3.1 of Anderson [2] that it is also regular.

In order to apply the results of Ferrari et al. [6], we relabel the states by defining $X(t)=$ $I(t)+(N+1) W(t)$, so that $\{X(t): t \geq 0\}$ is a Markov chain with state space $\{0,1,2, \ldots\}$, with state $x=0$ corresponding to the absorbing state $(i, w)=(0,0)$, and there is a one-to-one correspondence between states $(i, w) \in C$ and states $x \in\{1,2, \ldots\}$.

Defining $R=\inf \{t \geq 0: X(t)=0\}$, we first need to check the 'asymptotic remoteness' condition (1.4) of [6]. That is, we require that $\lim _{x \rightarrow \infty} \operatorname{Pr}(R<t \mid X(0)=x)=0$ for all $t \geq 0$. Now, if we start from any state $X(0)=x=i+(N+1) w$ then, before extinction can occur, it is necessary that all $w$ of the free-living infectious stages initially present either die or are consumed. The times until death or consumption of the free-living infectious stages are independent exponential random variables of mean $(\mu+v)^{-1}$; hence, for any $t>0$, it follows that

$$
\begin{aligned}
\operatorname{Pr}(R<t \mid X(0)=x) & \leq\left(1-\mathrm{e}^{-(\mu+v) t}\right)^{w} \\
& \rightarrow 0 \text { as } w \rightarrow \infty
\end{aligned}
$$

as required.

From Theorem 1.1 of [6], if asymptotic remoteness holds then a sufficient condition for the existence of a quasi-stationary distribution is that $\mathrm{E}\left[\mathrm{e}^{\theta R} \mid X(0)=x\right]<\infty$ for some $\theta>0$ and some $x \geq 1$. Furthermore, Ferrari et al. [6] showed, in their Lemma 4.3, that this condition is implied by their conditions (1.9)-(1.13). Essentially, we must find a suitably well-behaved nonnegative function $f(x)$ such that the process $f\left(X_{t}\right)$ has negative drift bounded away from 0 (except possibly when $X_{t}<D_{6}$ for some integer $D_{6}<\infty$ ) and is sufficiently well behaved for $X_{t}<D_{6}$.

For our SIS/W process, we can take $f(x)=w$ for $x=i+(N+1) w$. In condition (1.10) of [6], there appears to be a slight misprint, in that the transition rates $q(x, y)$ should be replaced by the transition probabilities $p(x, y)=q(x, y) /(-q(x, x))$ of the embedded discrete-time jump chain. With this correction, we then require that, for some $D_{1}>0$ and some integer $D_{6}<\infty$,

$$
\sum_{y \neq x} p(x, y) f(y) \leq f(x)-D_{1} \quad \text { for } x \geq D_{6} .
$$

For our SIS/W model, recalling that $x=i(N+1) w$, with $f(x)=w$ we have from (1.1)(1.5) that

$$
\begin{aligned}
\sum_{y \neq x} p(x, y) f(y)-f(x) & =\frac{\lambda i-(\mu+v) w}{-q(x, x)} \\
& \leq \frac{\lambda N-(\mu+v) w}{(\beta+\gamma+\lambda) N+(\mu+v) w} \quad \text { for } w \geq \frac{\lambda N}{\mu+v} \\
& \rightarrow-1 \quad \text { as } x \rightarrow \infty,
\end{aligned}
$$

and, so, by taking any $D_{1}$ with $0<D_{1}<1$, a suitable integer $D_{6}<\infty$ can be found and condition (2.2) is satisfied.

Conditions (1.9) and (1.11)-(1.13) of [6] are now straightforward to verify and, by applying Lemma 4.3 and Theorem 1.1 of [6], we thus establish the existence of a quasi-stationary distribution for our SIS/W model (provided that $\beta, \gamma, \lambda, \mu, v, p, N>0$ ). 
The existence of a quasi-stationary distribution is a necessary condition for the existence of a limiting conditional distribution, and, if the initial distribution of our process is taken to equal the quasi-stationary distribution, then clearly the limiting conditional distribution will also equal the quasi-stationary distribution. However, note that the existence of a quasi-stationary distribution does not guarantee the existence of the limiting conditional distribution of the process starting from some fixed initial state $(i, w)$ (see [13]).

\section{Normal approximation and the effect of indirect transmission}

We now approximate our SIS/W model using a diffusion approximation developed by Kurtz [8], [9]. We will find it more convenient to refer to the more specialized versions of Kurtz's main theorems which appeared in [14]. Regarding our SIS/W model as a densitydependent process, we define a family of scaled processes, indexed by $N$, by

$$
V_{N}(t)=\frac{1}{N}\left(I_{N}(t), W_{N}(t)\right)^{\top} .
$$

Consider the limit as $N \rightarrow \infty$ of $\boldsymbol{V}_{N}(t)$, with $\beta, \gamma, \lambda, \mu, v$, and $p$ held constant as $N$ varies. Setting $\boldsymbol{v}^{*}=\left(\hat{\imath}^{*}, \hat{w}^{*}\right)^{\top}$, where $\hat{\imath}^{*}$ and $\hat{w}^{*}$ are given by (1.7), it follows from Theorem 3.2 of [14] that, provided $\lim _{N \rightarrow \infty} \sqrt{N}\left(\boldsymbol{V}_{N}(0)-\boldsymbol{v}^{*}\right)=\boldsymbol{z}$ exists, the sequence of processes $\boldsymbol{Z}_{N}(\cdot)=$ $\sqrt{N}\left(\boldsymbol{V}_{N}(\cdot)-\boldsymbol{v}^{*}\right)$ converges weakly, in the space of all sample paths on any given finite time interval, to a bivariate Ornstein-Uhlenbeck process $\boldsymbol{Z}(\cdot)$ starting at $\boldsymbol{Z}(0)=\boldsymbol{z}$ with local drift matrix

$$
\boldsymbol{B}=\left(\begin{array}{cc}
-\beta \hat{\imath}^{*}-\frac{p v \lambda}{\mu+v} & p v\left(1-\hat{\imath}^{*}\right) \\
\lambda & -(\mu+v)
\end{array}\right)
$$

and local covariance matrix

$$
\boldsymbol{G}=\left(\begin{array}{cc}
2 \gamma \hat{\imath}^{*} & -\frac{p \nu \lambda}{\mu+v} \hat{\imath}^{*}\left(1-\hat{\imath}^{*}\right) \\
-\frac{p \nu \lambda}{\mu+v} \hat{\imath}^{*}\left(1-\hat{\imath}^{*}\right) & 2 \lambda \hat{\imath}^{*}
\end{array}\right) .
$$

This Ornstein-Uhlenbeck process has as its stationary distribution the bivariate normal distribution with zero mean vector and covariance matrix $\boldsymbol{\Sigma}$ satisfying

$$
\boldsymbol{B} \boldsymbol{\Sigma}+\boldsymbol{\Sigma} \boldsymbol{B}^{\top}=-\boldsymbol{G} .
$$

What this means in practice is that, for fixed $N$, by taking $\boldsymbol{z}=\sqrt{N}\left(\boldsymbol{V}_{N}(0)-\boldsymbol{v}^{*}\right)$, we can approximate the quasi-stationary distribution of our SIS/W model by a bivariate normal distribution with mean $\left(N \hat{\imath}^{*}, N \hat{w}^{*}\right)$ and covariance matrix $N \boldsymbol{\Sigma}$. For the approximation to be valid, we require that the step size $1 / N$ of the scaled process is sufficiently small for it to be approximated by a process with continuous sample paths; that the equilibrium point $\boldsymbol{v}^{*}=\left(\hat{\imath}^{*}, \hat{w}^{*}\right)^{\top}$ is stable, so that the process will tend to spend a long time in the vicinity of $\boldsymbol{v}^{*}$; and that both $\hat{\imath}^{*}$ and $\hat{w}^{*}$ are sufficiently large that the Ornstein-Uhlenbeck process is unlikely to reach the boundaries at $I=0$ and $W=0$. Thus, we expect the approximation to be good provided that the infection process is above threshold, in that $R_{0}>1$ and $N$ is sufficiently large. In the remainder of this section we investigate the approximating normal distribution, assuming it to provide a good approximation to the true quasi-stationary distribution; in Section 4 we will investigate the accuracy of the approximation numerically. 
A question of some practical interest is the effect of indirect, as opposed to direct, transmission on the quasi-stationary distribution of the process. Suppose that we allow the parameters to vary in such a way that $R_{0}$ remains fixed. That is, we keep the total infectivity of each individual constant, while varying the proportion of transmission that is indirect. Then $\hat{\imath}^{*}$ remains fixed, meaning that varying the proportion of transmission that is indirect does not affect the mean of the approximating normal distribution of infected hosts. However, $\hat{w}^{*}$ and the variance matrix $\boldsymbol{\Sigma}$ may be affected.

Specifically, by first writing $s=1-\hat{\imath}^{*}$ and then solving (3.1) for $\Sigma$ and eliminating $\beta$ using the substitution $\beta=\gamma / s-p \nu \lambda /(\mu+\nu)$, we find, after some tedious algebra, that (in obvious notation)

$$
\begin{aligned}
\sigma_{I}^{2} & =s\left(1-\frac{p v \lambda s^{2}}{D}\right), \\
\sigma_{W}^{2} & =\frac{\lambda(1-s)}{\mu+v}+\frac{\lambda^{2} s^{2}}{D}, \\
\sigma_{I W} & =\frac{\lambda(\mu+v) s^{2}}{D}
\end{aligned}
$$

where $D=\gamma(\mu+v)(1-s)+(\mu+v)^{2} s+p v \lambda s^{2}$.

First notice that $\sigma_{I}^{2} \leq s$, with equality when $p \nu \lambda=0$, which is when all transmission is via the direct route. Thus, any transmission via the indirect route results in a marginal quasistationary distribution for $I$ with smaller variance than if all transmission were via the direct route, assuming a fixed total infectivity per individual.

To investigate further the effect of indirect versus direct transmission, we consider the effect of varying any one of the parameters $p, \lambda, \mu$, or $\nu$, while simultaneously varying $\beta$ in such a way that $s$ (or, equivalently, $R_{0}$ ) remains constant. We find from (3.2) that

$$
\begin{aligned}
\frac{\mathrm{d}\left(\sigma_{I}^{2}\right)}{\mathrm{d} p} & =-(\gamma(1-s)+(\mu+v) s)(\mu+v) \frac{v \lambda s^{3}}{D^{2}}<0, \\
\frac{\mathrm{d}\left(\sigma_{I}^{2}\right)}{\mathrm{d} \lambda} & =-(\gamma(1-s)+(\mu+v) s)(\mu+v) \frac{p v s^{3}}{D^{2}}<0, \\
\frac{\mathrm{d}\left(\sigma_{I}^{2}\right)}{\mathrm{d} \mu} & =(\gamma(1-s)+2(\mu+v) s) \frac{p v \lambda s^{3}}{D^{2}}>0, \\
\frac{\mathrm{d}\left(\sigma_{I}^{2}\right)}{\mathrm{d} \nu} & =-\left(\gamma \mu(1-s)+\left(\mu^{2}-v^{2}\right) s\right) \frac{p \lambda s^{3}}{D^{2}} .
\end{aligned}
$$

An increase in indirect transmission corresponds to an increase in any one of $p, \lambda$, or $v$, or to a decrease in $\mu$. We see that increasing $p$ or $\lambda$, or decreasing $\mu$, leads to a decrease in $\sigma_{I}^{2}$. In the case of $\nu$, the situation is a little more complicated; increasing $v$ results in a decrease in $\sigma_{I}^{2}$ as long as $\gamma \mu(1-s)+\left(\mu^{2}-v^{2}\right) s>0$ or, equivalently, $v<\left(\mu^{2}+\gamma \mu(1-s) / s\right)^{1 / 2}$. In particular, if $v<\mu$ then $\mathrm{d}\left(\sigma_{I}^{2}\right) / \mathrm{d} v<0$. Thus, as long as the rate $v$, at which free-living infectious stages are consumed by the host population, is no greater than their natural death rate $\mu$, then any increase in the proportion of transmission via the indirect, rather than the direct, route results in a reduction in the variance of (our approximation to) the quasi-stationary distribution of $I$. It does not seem particularly obvious a priori that this should be the case, but may perhaps be intuitively understood as a result of the environmental reservoir of infection having a damping effect upon stochastic fluctuations of the process. 
Note that we would not generally expect to observe the behaviour of the free-living infectious stages directly, which is why we have focused on the marginal process $I$ describing the behaviour of the observed host population. A related point is that, because we only expect to observe the host population, $m_{i w}$ is not the only limiting conditional distribution of interest. An alternative is the limiting conditional distribution $\tilde{m}_{i w}$, defined by

$$
\tilde{m}_{i w}=\lim _{t \rightarrow \infty} \operatorname{Pr}((I(t), W(t))=(i, w) \mid I(s)>0 \text { for } 0 \leq s \leq t) .
$$

Whereas $m_{i w}$ describes the long-term behaviour of the process prior to total extinction of infection, $\tilde{m}_{i w}$ describes the long-term behaviour during a single observed outbreak in the host population. In Section 2 we proved the existence of a quasi-stationary distribution conditional upon nonextinction of infection within the combined population of hosts and free-living infectious stages. It is not clear how one would go about proving the existence of a quasi-stationary distribution conditional upon nonextinction of infection from the host population alone (corresponding to the limiting conditional distribution $\tilde{m}_{i w}$ ). There are an infinite number of states

$$
(i, w)=(1, w), \quad w=0,1,2, \ldots,
$$

all of which are adjacent to the set $\{(i, w): i=0\}$, so the asymptotic remoteness condition no longer holds and it seems that the methods of [6] are not applicable. On the other hand, assuming its existence, the bivariate normal distribution derived above should be equally useful as an approximation to either $m_{i w}$ or $\tilde{m}_{i w}$. This is because the Ornstein-Uhlenbeck process can only give a valid approximation provided that the probability of reaching the boundaries $I=0$ and $W=0$ is sufficiently small. We discuss the range of validity of the approximation further in the next section.

\section{Numerical results and persistence times}

All numerical work was carried out in MATLAB ${ }^{\circledR} 6.5$ running under Microsoft ${ }^{\circledR}$ Windows $^{\circledR}$ on a desktop personal computer.

In choosing parameter values for numerical work, we shall be guided by the work of Turner et al. [15] on E. coli O157 infection in dairy cattle. The model of [15] takes the total herd size to be 175 animals, the herd being split into four management groups of which the most important is the lactating group of around 95 animals. Thus, we shall take a host population size of $N=100$. It was stated in [15] that the infectious dose is 100 c.f.u. (colony forming units, or viable bacteria), so we reflect this by setting $p=0.01$. In the lactating group, the recovery rate is given as $\gamma=0.143$ (days) $^{-1}$ and the shedding rate as $\lambda=1.3 \times 10^{10}$ c.f.u. $(\text { cattle })^{-1}$ (days $)^{-1}$. The death rate of bacteria in the environment, in the absence of outside intervention, is given as $\mu=0.118$ (days) $^{-1}$. The transmission parameters $\beta$ and $\nu$ were described in [15] as 'unknown', and values then chosen to try to reflect knowledge of observed E. coli $\mathrm{O} 157$ epidemiology. We will choose values for $\beta$ and $v$ with the aim of understanding the behaviour of our SIS/W model, rather than to reflect a specific real-life infection. Note that the parameter sets used in this section all satisfy the condition $v<\mu$ of Section 3 .

When simulating using plausible parameter values, a problem arises in that the free-living infectious stages process $W(t)$ undergoes transitions at a much greater rate than infective hosts process $I(t)$ and, thus, to observe the development of the process $I(t)$ is extremely time consuming. Since the problem is that the $W(t)$ process makes a very large number of transitions in a small time, we can circumvent this difficulty by treating $W(t)$ as deterministic, 
at least between the transitions of $I(t)$. That is, we approximate our original SIS/W model by a piecewise-deterministic Markov process (see [5]) with the following transition rates:

$$
\begin{array}{lll}
I \rightarrow I+1 & \text { at rate } & (\beta / N) I(N-I)+(p v / N) W_{D}(N-I), \\
I \rightarrow I-1 & \text { at rate } & \gamma I .
\end{array}
$$

Here, $W_{D}(t)$ evolves deterministically between transitions of $I(t)$ according to

$$
\frac{\mathrm{d} W_{D}}{\mathrm{~d} t}=\lambda I-(\mu+v) W_{D}
$$

For the parameter values that we use, as long as any infective hosts are present, $W(t)$ will be of order $10^{11}$, which is certainly sufficiently large that the piecewise-deterministic Markov process described above is realistic.

Suppose that the $I(t)$ process makes a transition at time $T$, and that $\left(I(T), W_{D}(T)\right)=(i, w)$. Denote by $\tau$ the time until the next transition of $I(t)$. Then, solving (4.3) gives

$$
W_{D}(t)=w \mathrm{e}^{-(\mu+v) t}+\frac{\lambda}{\mu+v} i\left(1-\mathrm{e}^{-(\mu+v) t}\right), \quad T \leq t<T+\tau .
$$

It follows from (4.1) and (4.2) that $\tau$ has distribution function

$$
\begin{aligned}
\operatorname{Pr}(\tau \leq t)=1-\exp \{ & -\left(\left(\beta+\frac{p \lambda v}{\mu+v}\right)(N-i) \frac{i}{N}+\gamma i\right) t \\
& \left.-\frac{p v}{N(\mu+v)}(N-i)\left(w-\frac{\lambda}{\mu+v} i\right)\left(1-\mathrm{e}^{-(\mu+v) t}\right)\right\} .
\end{aligned}
$$

For $i>0$, it is straightforward to simulate from the distribution (4.4) of $\tau$ by rejection sampling, using an exponential proposal distribution with mean $M^{-1}$, where

$$
M=\left(\beta+\frac{p \lambda v}{\mu+v}\right)(N-i) \frac{i}{N}+\gamma i+\min \left\{0, \frac{p v}{N}(N-i)\left(w-\frac{\lambda}{\mu+v} i\right)\right\} .
$$

When $i=0$ we have $M=0$, meaning that the rejection sampling method breaks down; however, in this case we can simulate $\tau$ by inverting the distribution function (4.4), noting that, for $i=0$, there is a nonzero probability that $\tau=\infty$. To determine whether the event at time $T+\tau$ is an infection or a recovery, we observe that the probabilities of these two events are in the proportions given by (4.1) and (4.2), respectively, with $W_{D}$ evaluated at time $T+\tau$.

Note that our simulation process $\left(I(t), W_{D}(t)\right)$ can never reach a state with $W_{D}=0$ within finite time (unless $I(0)=W_{D}(0)=0$ ). Consequently, in our numerical work we shall focus upon the limiting conditional distribution $\tilde{m}_{i w}$, defined by (3.3), rather than $m_{i w}$, defined by (2.1). We shall also focus on the marginal distribution of the number of infected hosts $I$, as this is what we would expect to observe.

Since we expect the normal approximation of Section 3 to perform well for $R_{0}$ values sufficiently greater than the threshold value $R_{0}=1$, we first consider the situation well above threshold. Specifically, in order to obtain $R_{0}=2$ with direct and indirect transmissions making equal contributions to $R_{0}$, with $N=100, p=0.01, \gamma=0.143$ (days) $^{-1}, \lambda=$ $1.3 \times 10^{10}$ c.f.u. (cattle) ${ }^{-1}$ (days) $^{-1}$, and $\mu=0.118$ (days) $^{-1}$, we take $\beta=0.143{\text { (days })^{-1}}^{-1}$ and $v \approx 1.298 \times 10^{-10}$ (days) $)^{-1}$. We carried out 100000 simulation runs with these parameter values, each initiated at a point close to the deterministic endemic equilibrium point $\left(N \hat{\imath}^{*}, N \hat{w}^{*}\right)$ 


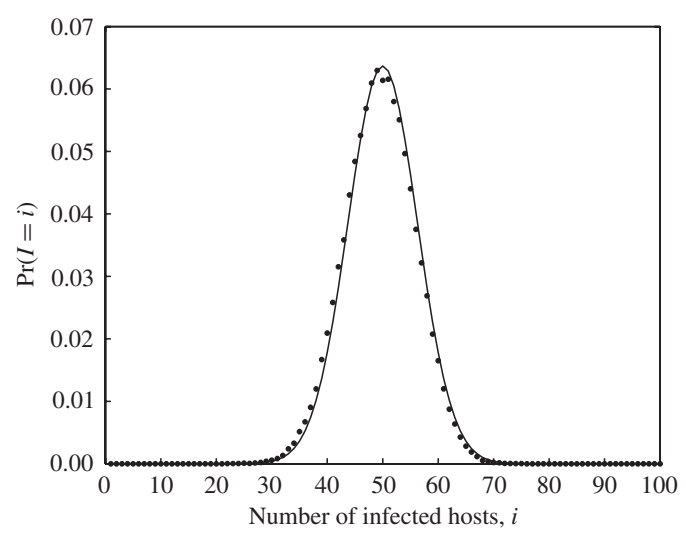

FIGURE 1: The simulated quasi-stationary distribution compared to the normal approximation: dots indicate the empirical distribution from simulation, while the smooth curve represents the normal approximation of Section 3. The parameter values are $N=100, p=0.01, \gamma=0.143$ (days) $^{-1}$, $\lambda=1.3 \times 10^{10}$ c.f.u. $(\text { cattle })^{-1}$ (days) $^{-1}, \mu=0.118$ (days) $^{-1}, \beta=0.143$ (days) $)^{-1}$, and $v \approx$ $1.298 \times 10^{-10}(\text { days })^{-1}$, meaning that $R_{0}=2$. Simulation results are from 100000 simulation runs, each initiated at a point close to the deterministic endemic equilibrium point $\left(N \hat{\imath}^{*}, N \hat{w}^{*}\right)$ and run until time $t=100$ days.

and run until time $t=100$ days (and similarly for all subsequent simulation results). The results are shown in Figure 1, in which the dots indicate the simulated empirical distribution and the smooth curve represents the normal approximation of Section 3. We see that our normal approximation does indeed perform well for these parameter values. No simulation runs became extinct in the host population $(I=0)$ within 100 days.

To investigate the effect of indirect versus direct transmission when the process is well above threshold, we again take $R_{0}=2$, but now consider the two extreme cases

$$
\beta=0.286(\text { days })^{-1}, \quad v=0(\text { days })^{-1}
$$

(direct transmission only) and

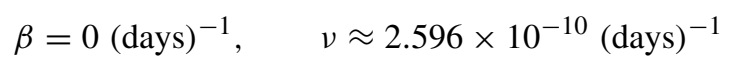

(indirect transmission only), with the other parameter values as before. For indirect transmission only, we used simulation to evaluate the quasi-stationary distribution. With direct transmission only, the process $I(t)$ is precisely the classical SIS infection model, a Markov process on the finite state space $\{0,1, \ldots, N\}$. Consequently, the quasi-stationary distribution of $I$ can be computed exactly. Denoting by $Q$ the transition rate matrix of the SIS infection process, and by $\boldsymbol{Q}_{C}$ the matrix $\boldsymbol{Q}$ truncated by deleting the row and column corresponding to the absorbing state $i=0$, the quasi-stationary distribution of $I$ is given by the left eigenvector of $\boldsymbol{Q}_{C}$ corresponding to the eigenvalue of maximal real part, normalized such that its components sum to 1 . (The distribution computed in this way corresponds to $\tilde{m}_{i w}$ rather than $m_{i w}$.)

The results are shown in Table 1. These numerical results confirm that direct transmission leads to greater variability than indirect transmission and that the approximating formula $\sigma_{I} \sqrt{N}$, where $\sigma_{I}$ is given by (3.2), gives a reasonably good idea of the extent of the difference. Graphs corresponding to Figure 1 for the cases $\beta=0.286$ (days) $^{-1}, v=0$ (days) $^{-1}$ and $\beta=$

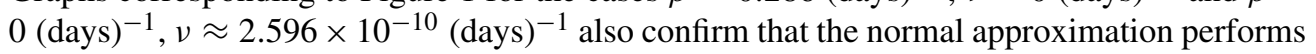


TABLE 1: Mean and standard deviation (SD) values for the quasi-stationary distribution of the number of infected hosts $I$, and approximating formulae from Section 3. The quasi-stationary distribution was computed by the eigenvector method in the cases with direct transmission only $\left.(v=0 \text { (days })^{-1}\right)$, and by simulation (100000 runs until time $t=100$ days) in the cases with indirect transmission only

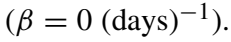

\begin{tabular}{lllccccc}
\hline & & & \multicolumn{2}{c}{ Simulation/Eigenvector } & & \multicolumn{2}{c}{ Normal approximation } \\
\cline { 6 - 7 }$R_{0}$ & $\beta\left((\text { days })^{-1}\right)$ & $v\left((\text { days })^{-1}\right)$ & Mean & SD & & Mean $=N \hat{\imath}^{*}$ & SD $=\sigma_{I} \sqrt{N}$ \\
\hline 2 & 0.286 & 0 & 48.93 & 7.23 & & 50 & 7.07 \\
2 & 0 & $2.596 \times 10^{-10}$ & 49.69 & 5.72 & & 50 & 5.68 \\
1.25 & 0.17875 & 0 & 16.89 & 8.63 & & 20 & 8.94 \\
1.25 & 0 & $1.623 \times 10^{-10}$ & 18.78 & 6.37 & & 20 & 6.44 \\
\hline
\end{tabular}

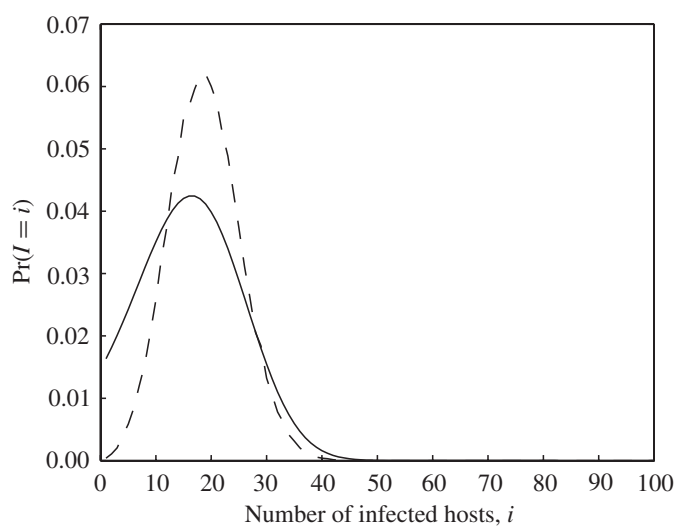

FIGURE 2: Quasi-stationary distributions comparing direct with indirect transmission: the continuous

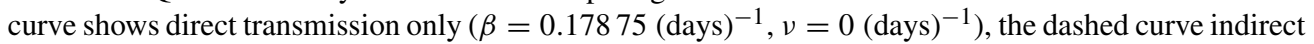
transmission only $\left.(\beta=0 \text { (days })^{-1}, v \approx 1.623 \times 10^{-10}(\text { days })^{-1}\right)$. In each case, $N=100, p=0.01$,

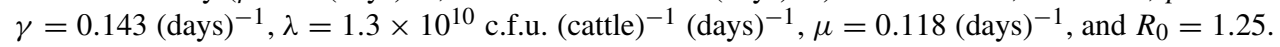

reasonably well, although it appears a little less accurate in the case that all transmission is via the direct route. (These graphs are quite similar to Figure 1, and are omitted.)

If the process is not well above threshold, we would not expect our normal approximation to perform so well. Thus, we next consider the case $R_{0}=1.25$, looking at the two extremes of direct transmission alone versus indirect transmission alone. To obtain $R_{0}=1.25$, we take either $\beta=0.17875$ (days) $^{-1}, v=0$ (days) $^{-1}$ (direct transmission only) or $\beta=0$ (days) $^{-1}$, $v \approx 1.623 \times 10^{-10}$ (days) $^{-1}$ (indirect transmission only). The other parameter values are as before. Figure 2 shows the computed marginal quasi-stationary distributions of the number of infected hosts $I$; mean and standard deviation values are presented in Table 1.

With indirect transmission only, the normal approximation again performs reasonably well, in terms of both the shape of the distribution and the mean and standard deviation values. With direct transmission only, however, it is clear from Figure 2 that our normal approximation will perform considerably less well, since the computed quasi-stationary distribution is noticeably truncated at $I=0$. We see from Table 1 that, although the standard deviation estimate for this case is reasonably close to the true value, the mean is significantly overestimated. 
Nåsell [11], with reference to the classical SIS infection model, suggested as a rule of thumb that the normal approximation of the quasi-stationary distribution tends to perform well when $\hat{\imath}^{*}>0$ and the coefficient of variation, $C V_{I}=\sigma_{I} / \hat{\imath}^{*} \sqrt{N}$, satisfies $C V_{I}<\frac{1}{3}$. This is because a normally distributed random variable takes values within three standard deviations of its mean with high probability, so that, provided $C V_{I}<\frac{1}{3}$, the probability assigned to negative $I$ values by the approximation is small. To be confident that our bivariate normal approximation for the SIS/W model will perform well, we require both $C V_{I}<\frac{1}{3}$ and $C V_{W}<\frac{1}{3}$, where $C V_{W}=\sigma_{W} / \hat{w}^{*} \sqrt{N}$. All of the above simulation results satisfy this except for the case

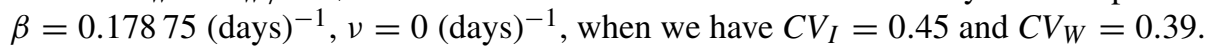

All the results of the eigenvector method were checked by simulation (100000 runs until $t=100$ days in each case), starting from a fixed state close to the deterministic equilibrium point $\left(N \hat{\imath}^{*}, N \hat{w}^{*}\right)$. All of the simulation results were conditioned on $I(t)>0$ for $0 \leq t \leq 100$. With $R_{0}=2$, all the simulation runs satisfied $I(t)>0$ for $0 \leq t \leq 100$. With $R_{0}=1.25$, within 100 days there were 169 extinctions of infection from the host population in the case of indirect

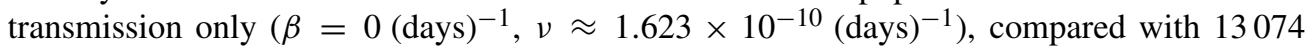

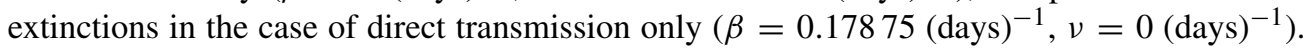
This suggests that indirect transmission may tend to increase persistence of infection, compared to direct transmission.

In fact, the results of Section 3 are of particular interest with regard to persistence times. When $\sigma_{I} \sqrt{N}$ is large relative to $N \hat{\imath}^{*}$, the quasi-stationary distribution of $I$ attaches substantial probability to $I$ values close to 0 , meaning that infection tends to die out of the host population quickly. More specifically, the time to extinction of an outbreak from the host population, for a process initiated from the quasi-stationary distribution $\tilde{m}_{i w}$, is exponentially distributed with constant hazard rate $\gamma \sum_{w} \tilde{m}_{1 w}$. (Similarly, the time to complete extinction of infection, for a process initiated from the quasi-stationary distribution $m_{i w}$, is exponentially distributed with constant hazard rate $\gamma m_{10}+(\mu+v(1-p)) m_{01}$. $)$ As an explicit formula for $\sum_{w} \tilde{m}_{1 w}$ is not available, a simple alternative measure is given by $C V_{I}$. Large values of $C V_{I}$ suggest that outbreaks will die out quickly, whereas small values suggest likely long-term persistence in the host population. Returning to our numerical results for the case $R_{0}=1.25$, we find that in the case $\beta=0.17875$ (days) $^{-1}, v=0$ (days) $^{-1}$, we have $C V_{I}=0.45$, whereas with $\beta=0$ (days) $^{-1}, v \approx 1.623 \times 10^{-10}$ (days) $^{-1}$, we have $C V_{I}=0.32$. The estimate of expected time to outbreak extinction starting from quasi-stationarity $\left(\left(\gamma \sum_{w} \tilde{m}_{1 w}\right)^{-1}\right)$ is found to be 436 days in the case $\beta=0.17875$ (days) $^{-1}, v=0$ (days) $^{-1}$, compared with 17900 days in the case $\beta=0$ (days) $)^{-1}, v \approx 1.623 \times 10^{-10}$ (days) $^{-1}$. Thus, transmission via the indirect route can lead to much longer outbreaks of infection in the host population than direct transmission, for the same $R_{0}$ value. In many deterministic infection models, long-term endemic persistence is predicted precisely when $R_{0}>1$; here, we see clearly that, when stochastic effects are taken into account, the value of $R_{0}$ is not in itself sufficient to determine whether persistence is likely.

In the classical SIS infection model, Andersson and Djehiche [3] have studied persistence times in some detail; the case in which $R_{0}$ is close to 1 has been more fully investigated by Nåsell [11]. Their results indicate that $C V_{I}$ does not give a very precise estimate of the expected time to extinction; nonetheless, $C V_{I}$ does at least give a rough idea of whether outbreaks are likely to persist in the long term, based on a simple explicit formula.

\section{Acknowledgements}

It is a pleasure to thank Pablo Ferrari, Sang Taphou Mendy, and Yanni Xiao for helpful remarks. 


\section{References}

[1] Anderson, R. M. and May, R. M. (1981). The population dynamics of microparasites and their invertebrate hosts. Phil. Trans. R. Soc. London B 291, 451-524.

[2] Anderson, W. J. (1991). Continuous-Time Markov Chains. An Applications-Oriented Approach. Springer, Berlin.

[3] Andersson, H. and Djehiche, B. (1998). A threshold limit theorem for the stochastic logistic epidemic. J. Appl. Prob. 35, 662-670.

[4] Clancy, D., O’Neill, P. D. And Pollett, P. K. (2001). Approximations for the long-term behaviour of an open-population epidemic model. Methodology Comput. Appl. Prob. 3, 75-95.

[5] Davis, M. H. A. (1993). Markov Models and Optimization. Chapman and Hall, London.

[6] Ferrari, P., Kesten, H., Martínez, S. and Picco, P. (1995). Existence of quasi-stationary distributions. A renewal dynamic approach. Ann. Prob. 23, 501-521.

[7] Kijima, M., Nair, M. G., Pollett, P. K. and van Doorn, E. A. (1997). Limiting conditional distributions for birth-death processes. Adv. Appl. Prob. 29, 185-204.

[8] Kurtz, T. G. (1970). Solutions of ordinary differential equations as limits of pure jump Markov processes. J. Appl. Prob. 7, 49-58.

[9] KurTz, T. G. (1971). Limit theorems for sequences of jump Markov processes approximating ordinary differential processes. J. Appl. Prob. 8, 344-356.

[10] NÅsell, I. (1996). The quasi-stationary distribution of the closed endemic SIS model. Adv. Appl. Prob. 28, 895-932.

[11] NÅSELl, I. (1999). On the quasi-stationary distribution of the stochastic logistic epidemic. Math. Biosci. 156, 21-40.

[12] NÅSELl, I. (1999). On the time to extinction in recurrent epidemics. J. R. Statist. Soc. B 61, 309-330.

[13] PAKES, A. G. (1995). Quasi-stationary laws for Markov processes: examples of an always proximate absorbing state. Adv. Appl. Prob. 27, 120-145.

[14] Pollett, P. K. (1990). On a model for interference between searching insect parasites. J. Austral. Math. Soc. Ser. B 31, 133-150.

[15] Turner, J., Begon, M., Bowers, R. G. And French, N. P. (2003). A model appropriate to the transmission of a human food-borne pathogen in a multigroup managed herd. Prev. Vet. Med. 57, 175-198.

[16] Weiss, G. H. and Dishon, M. (1971). On the asymptotic behaviour of the stochastic and deterministic models of an epidemic. Math. Biosci. 11, 261-265.

[17] Xiao, Y., Bowers, R. G., Clancy, D. and French, N. P. (2005). Dynamics of infection with multiple transmission mechanisms in unmanaged/managed animal populations. Submitted. 